\title{
Plasma efavirenz concentration inversely correlates with increased risk of cytomegalovirus infection in HIV- infected pregnant women
}

\author{
D Mhandire, ${ }^{1,2}$ MPhil; G Morse, ${ }^{3}$ PharmD; C Maponga, ${ }^{4}$ PharmD; K Mhandire, ${ }^{1,2,5} \mathrm{PhD}$; C Dandara, ${ }^{1,2} \mathrm{PhD}$ \\ ${ }^{1}$ Division of Human Genetics, Department of Pathology, Faculty of Health Sciences, University of Cape Town, South Africa \\ ${ }^{2}$ Institute of Infectious Disease and Molecular Medicine, Faculty of Health Sciences, University of Cape Town, South Africa \\ ${ }^{3}$ Translational Pharmacology Research Core, New York State Center of Excellence in Bioinformatics and Life Sciences, New York, NY, USA \\ ${ }^{4}$ School of Pharmacy, University of Zimbabwe, Harare, Zimbabwe \\ ${ }^{5}$ Department of Chemical Pathology, College of Health Sciences, University of Zimbabwe, Harare, Zimbabwe
}

Corresponding author: C Dandara (collet.dandara@uct.ac.za)

Background. Effective combination antiretroviral therapy (cART) has tremendously reduced HIV-associated morbidity, mortality and mother-to-child transmission. However, the benefits of cART are threatened by comorbidities, adverse drug reactions and virus resistance to existing treatment regimens. One of the most occurring comorbidities is cytomegalovirus (CMV) infection.

Objectives. To investigate the effects of cART on the occurrence of CMV infection among pregnant women.

Methods. Using a cross-sectional study design, 175 HIV-infected pregnant women were recruited, and data were obtained from their clinical records. Blood samples were collected for host DNA, CMV DNA and plasma efavirenz (EFV) measurement. CMV DNA was measured using real-time polymerase chain reaction (PCR). CYP2B6 c.516G>T and CYP2B6 c.983T>C single nucleotide polymorphisms were characterised using PCR/restriction fragment length polymorphism and TaqMan assays, respectively. Plasma EFV concentrations were determined using high-performance liquid chromatography.

Results. There was an inverse association between plasma EFV concentration and CMV DNA. Participants with lower plasma EFV concentrations were significantly $(p<0.001)$ more likely to be CMV DNA positive than those with higher plasma concentrations. This result is also supported by the observation that carriers of CYP2B6 poor-metaboliser genotypes (CYP2B6 c.516T/T and CYP2B6 c.983T/C) were less likely to be positive for CMV DNA. Furthermore, poor metabolism as denoted by CYP2B6 c.516T/T and CYP2B6 c.983T/C genotypes was significantly associated with lower CMV viral load.

Conclusions. HIV treatment disrupts the balance between host and co-infecting microbes. Reduced or subtherapeutic levels of antiretroviral drugs, which could be exacerbated by genetic polymorphisms in drug metabolism genes and non-adherence, predispose infected individuals to an increased risk of CMV infection in pregnancy.

S Afr Med J 2020;110(1):10-15. https://doi.org/10.7196/SAMJ.2020.v110i1.14316

Combination antiretroviral therapy (cART) has tremendously reduced HIV-associated morbidity, mortality and mother-to-child transmission (MTCT). ${ }^{[1]}$ The Option B+ (lifelong cART for all HIV-infected pregnant and breastfeeding women) introduced by the World Health Organization proved to be effective, resulting in a reduction of MTCT from $33 \%$ (before ART) to $<2 \%$ in subSaharan Africa. ${ }^{[2]}$ Despite the massive success in reduction of MTCT, HIV-exposed uninfected (HEU) children experience delayed developmental milestones, disabilities and more frequent morbidities than their HIV-unexposed counterparts. ${ }^{[3,4]}$ Developmental and health problems in some HEU infants occur despite maternal cART during pregnancy. Differential HIV outcomes have previously been associated with interindividual variation in response to cART. ${ }^{[5]}$

The differential outcomes of cART during pregnancy are critical in the care of HEU infants who have to endure effects of in utero and postpartum exposure to antiretroviral drugs. ${ }^{[6]}$ Furthermore, the increasing number of HIV-infected adolescents who grow into adulthood with a strong will to procreate, further emphasises the need to understand the factors associated with different HIV treatment outcomes in pregnancy, which also determine infant outcomes. ${ }^{[7]}$ Of the utmost importance during pregnancy are antenatal diseases that may result in adverse outcomes if transmitted to the developing fetus or neonate. Antenatal infections (such as cytomegalovirus (CMV), toxoplasmosis, syphilis and rubella) are more prevalent among HIVinfected than HIV-uninfected women. ${ }^{[8,9]}$

$\mathrm{CMV}$ is a latent beta herpesvirus that is often reactivated in immunecompromised populations such as HIV patients and pregnant women. When reactivation of CMV occurs during pregnancy, the virus can be transmitted to the fetus and/or neonate, resulting in congenital CMV (cCMV) infection. ${ }^{[10]}$ Effects of cCMV may be potentially fatal and include paediatric pneumonia, neurocognitive developmental delay and sensorineural hearing loss, for which CMV is the leading non-genetic cause. ${ }^{[11,12]}$ In a review by Filteau and Rowland-Jones, ${ }^{[13]}$ vertical transmission of CMV in utero or in early infancy was hypothesised to be responsible for the health and developmental deficits in HIV-exposed children. cCMV is sustained by antenatal $\mathrm{CMV}$ infection or reactivation; hence, controlling maternal CMV infection is key in the prevention of cCMV. cART is protective against the emergence of opportunistic infections; ${ }^{[14}$ therefore, the occurrence of CMV in some but not all HIV-infected 
pregnant women could suggest differences in protectivity of cART against co-infections.

In resource-limited settings, the choice of cART Option B+ is a combination of two nucleoside reverse transcriptase inhibitors (NRTIs) (tenofovir (TDF) and lamivudine (3TC)/emtricitabine (FTC)) and one non-nucleoside reverse transcriptase inhibitor (NNRTI) (efavirenz (EFV)). The use of EFV-based regimens among pregnant women has been recommended, as EFV has fewer drug interactions and fewer adverse drug reactions than other antiretroviral drugs. ${ }^{[15]}$ Therefore, EFV is the NNRTI of choice for first-line treatment of HIV infection in pregnancy.

EFV is administered as a once-daily oral dose of $600 \mathrm{mg}$ and is mainly metabolised by cytochrome P450 2B6 (CYP2B6), a hepatic enzyme encoded by the CYP2B6 gene. ${ }^{[16]}$ However, genetic polymorphisms in CYP2B6 affect the pharmacokinetics of EFV, leading to variability in EFV steady-state concentrations. ${ }^{[17,18]}$ Participants in this study were administered EFV-based cART regimens; therefore, EFV will be used as a proxy for cART in this report on susceptibility to CMV infection during pregnancy. The therapeutic effect of a drug is influenced by its rate of metabolism and excretion, which can be approximated by the plasma concentration of the drug. ${ }^{[4,18]}$ Two single nucleotide polymorphisms CYP2B6 c.516G>T (rs3745274) and CYP2B6 c.983T>C (rs28399499) in the CYP2B6 affect EFV metabolism. ${ }^{[19]}$ Moreover, we also investigated associations between plasma EFV concentration and CMV infection. CYP2B6 genotypes were also characterised to confirm their effects on variable plasma EFV concentrations.

\section{Methods \\ Study participants}

In this cross-sectional study, HIV-infected pregnant women were enrolled through the University of Zimbabwe College of Health Sciences birth cohort study. These women were receiving TDF, 3TC and EFV $600 \mathrm{mg}$, prescribed as part of their routine clinical care and for prevention of MTCT (PMTCT). Inclusion criteria were: gestational age $\geq 20$ weeks, maternal age $>18$ years, HIV infected on cART, and presenting for routine antenatal care at any one of three council polyclinics in the high-density suburbs of Kuwadzana, Dzivarasekwa and Glen View in Harare, Zimbabwe. The HIV status of the participants was obtained from clinic records, but additional confirmatory HIV testing was done at the time of sample collection. ${ }^{[20]}$ CMV DNA status and CMV viral loads were determined by real-time polymerase chain reaction (RT-PCR) using a RealStar CMV PCR Kit 1.0 (Altona Diagnostics, Germany), according to the manufacturer's instructions. Results for CMV status and CMV viral loads were available from the main cohort records.

\section{Ethical approval}

This study received ethical clearance from the Medical Research Council of Zimbabwe (ref. no. MRCZ/A/2177) and the University of Cape Town Institutional Review Board (ref. no. HREC628/2017). The study was carried out in accordance with the guidelines of the Helsinki Declaration of 2008 ethics clearance. Written informed consent was obtained from each participant prior to data and sample collection. A questionnaire was used to collect participant demographical information, and clinical parameters were obtained from the participants' medical records. A $5 \mathrm{~mL}$ ethylenediaminetetraacetic acid (EDTA) anticoagulated blood sample was collected from each participant at least 8 hours after administering a dose. Plasma was isolated from $3 \mathrm{~mL}$ of the whole blood sample (centrifugation at $3000 \mathrm{~g}$ for 10 minutes) within 12 hours of venepuncture and stored at $-80^{\circ} \mathrm{C}$ for $\mathrm{EFV}$ concentration measurement, while the remainder of the sample was used for DNA isolation.

\section{Determination of plasma efavirenz concentration and CYP2B6 genotypes}

Plasma EFV concentrations were quantified at the University of Zimbabwe-International Pharmacology Specialty Laboratory (UZ-IPSL) using high-performance liquid chromatography (HPLC). A photodiode array detector scanning at $247 \mathrm{~nm}$ wavelength was used for quantification of EFV. The assay had a lower limit of quantitation of $500 \mathrm{ng} / \mathrm{mL}$ with an interday imprecision coefficient of variance (CV) ranging from $1.0 \%$ to $5.6 \%$, as determined using the quality-control samples. The method used for EFV quantification was validated and approved by Clinical Pharmacology Quality Assurance (CPQA), an external quality assurance body. UZ-IPSL participates in the CPQA annual proficiency testing.

One of the factors that could affect plasma EFV is genetic variation in the CYP2B6 gene. The CYP2B6 gene encodes for the enzyme principally involved in the metabolism of EFV, and two polymorphisms (CYP2B6 c.516G $>T$ and $C Y P 2 B 6$ c.983T $>C$ ) have been shown to affect steady-state EFV exposure. Therefore, the contribution of CYP2B6 genetic variation in EFV therapeutic effects and risk of CMV acquisition needed to be determined. Host genomic DNA was extracted from whole blood using the Quick-DNA Miniprep Plus Kit (Zymo Research, USA) according to manufacturer's instructions. We genotyped for CYP2B6 c.516G $>T$ using restriction fragment length polymorphism. ${ }^{[21]}$ CYP2B6 c.983T $>C$ was genotyped using real-time allelic discrimination PCR on the BioRad CFX96 (Bio-Rad Laboratories, USA). TaqMan SNP Genotyping Assay and TaqMan Universal Master Mix were used to genotype CYP2B6 c.983T >C (rs28399499; assay ID C_4362691_10). All components were purchased from Thermo Fisher Scientific, USA, and genotyping was done according to manufacturer's instructions. Genotyping of single nucleotide polymorphisms (SNPs) was done at the Division of Human Genetics, Faculty of Health Sciences, University of Cape Town, South Africa.

\section{Statistical analysis}

Data were compiled and managed in Research Electronic Data Capture (REDCap) ${ }^{[22]}$ Statistical analyses were performed using Stata SE, version 15 (StataCorp, USA). Numerical variables were described as either median with interquartile range (IQR) for non-parametric variables or mean with standard deviation (SD) for parametric variables. Categorical variables were described as frequencies. Demographic and clinical characteristics were compared between CMV-infected and CMV-uninfected participants using $t$-tests for parametric data, Mann-Whitney tests for non-parametric data and $\chi^{2}$ tests for categorical data. Plasma EFV concentrations and HIV viral load were compared among participants based on CMV DNA status using the Mann-Whitney test. The $\chi^{2}$ test was used to determine the association between CMV infection status and EFV therapeutic groups. The relationship between CMV DNA load and HIV viral load was determined using Spearman's correlation coefficient test. Multivariate logistic regression was then performed to control for confounders such as maternal age, gestational age, gravidity, body mass index (BMI) and CD4+ count. Plasma EFV concentrations were compared among CYP2B6 c.516G>T genotypes using the KruskalWallis rank sum test and between CYP2B6 c.983T $>C$ genotypes using the Mann-Whitney test. The correlation between EFV concentration and plasma HIV viral load was determined using Spearman's correlation coefficient. Statistical significance was set at $p<0.05$. 


\section{Results}

Demographic and clinical characteristics of participants The demographic characteristics of the study participants are presented in Table 1. A total of $175 \mathrm{HIV}$-infected pregnant women who were receiving EFV-containing first-line cART were enrolled into the study. EFV concentration values were present in all 175 participants. CYP2B6 c.516G $>$ T and CYP2B6 c.983T >C SNP genotyping was also successfully done. All participants were in the reproductive age group (mean (range) $30(18$ - 44) years) and had been receiving EFV-based cART from a minimum of 2 months to a maximum of 140 months. The mean (SD) CD4+ cell count was $358(215) \mu \mathrm{L}$, while the median (25th - 75th percentile) HIV viral load was 97 (10 - 400) copies/mL. Results for CMV DNA status of the participants were available from the main study records. Of the 175 study participants, 27 (15.4\%) had CMV DNA detected and quantified in their plasma. The median (range) CMV viral load was 366 (108 - 103 355) copies/mL.

\section{Plasma efavirenz concentration distribution}

Plasma EFV concentrations were determined for all 175 participants from blood drawn at least 8 hours post dose. Participants selfreported good adherence (did not miss $>1$ dose since pregnancy). The median (25th - 75th percentile) plasma EFV concentration for this cohort of pregnant women was 1850 (990 - 3 963) ng/mL. The plasma EFV concentrations ranged from 250 to $15931 \mathrm{ng} / \mathrm{mL}$, exhibiting interindividual variability among patients despite them receiving the same $600 \mathrm{mg}$ daily EFV dose. Based on previously described therapeutic ranges, ${ }^{[23]}$ plasma EFV concentrations were assigned to three groups: subtherapeutic $(<1000 \mathrm{ng} / \mathrm{mL})$, therapeutic (1 $000-4000 \mathrm{ng} / \mathrm{mL}$ ) and supratherapeutic (>4 $000 \mathrm{ng} / \mathrm{mL})$ range. The distribution of plasma EFV concentration among participants according to categories was as follows: $44(25 \%)$ in the subtherapeutic range, $90(51 \%)$ in the therapeutic range, and 41 (24\%) in the supratherapeutic range.

\section{Association between CMV DNA positivity and plasma efavirenz concentration}

CMV DNA-positive women may be at risk of transmitting CMV to their offspring with potentially debilitating effects - with a greater risk of transmission in HIV-infected women. We investigated the possible role of plasma EFV concentration and consequently HIV disease progression (determined by HIV viral load) on maternal plasma CMV DNA status in participants who were HIV-CMV co-infected. CMV DNA was detected in the plasma of 27 participants (15.4\%).

The association between plasma EFV concentration and plasma CMV DNA positivity was determined using the Mann-Whitney test. Fig. 1 shows the effects of plasma EFV concentration on CMV DNA positivity. Reduced plasma EFV concentration (median (25th - 75th percentile) $847(250-3307) \mathrm{ng} / \mathrm{mL}$ ) was significantly associated with CMV DNA positivity $(p<0.001)$ compared with the higher plasma EFV concentration (median (25th - 75th percentile) 2024 (250 $14039) \mathrm{ng} / \mathrm{mL}$ ). Using the $\chi^{2}$ test, we found a significant decrease $(p<0.001)$ in the proportion of CMV positivity and plasma EFV concentration groups. The distribution of CMV DNA positivity in the three plasma EFV concentration groups was as follows: subtherapeutic (76\%), therapeutic (16\%) and supratherapeutic (8\%). Furthermore, using Spearman's correlation test, plasma concentrations inversely correlated with CMV viral load. Log plasma EFV concentration significantly decreased with increasing CMV viral load ( $r=-0.4 ; p=0.03$ ).

Using the Mann-Whitney test, CMV DNA positivity was significantly associated $(p<0.001)$ with higher HIV viral load (median

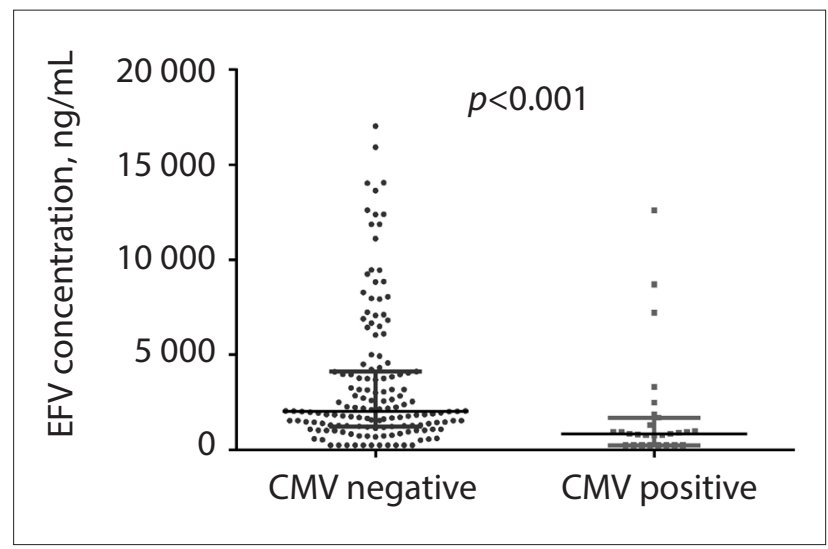

Fig. 1. Comparison of plasma EFV concentration between CMV-infected and CMV-uninfected participants. Data are presented with median and interquartile ranges. ( $E F V=$ efavirenz; $C M V=$ cytomegalovirus.)

Table 1. Participants' demographic and clinical characteristics

\begin{tabular}{|c|c|c|c|c|}
\hline Characteristic & Participants, $N=175$ & CMV negative, $n=148$ & CMV positive, $n=27$ & $p$-value \\
\hline \multicolumn{5}{|l|}{ Demographics } \\
\hline Mean (SD) maternal age, years & $30(6)$ & $30(6)$ & $29(6)$ & $0.182^{\dagger}$ \\
\hline Mean (SD) gestational age, weeks & $32(5)$ & $32(5)$ & $32(4)$ & $0.945^{\dagger}$ \\
\hline Mean (SD) gravidity & $3(1)$ & $3(1)$ & $3(2)$ & $0.285^{\dagger}$ \\
\hline Mean (SD) parity & $1.7(1)$ & $2(1)$ & $1(1)$ & $0.052^{\dagger}$ \\
\hline Median (25th - 75th) BMI, kg/m² & $29(23-28)$ & $26(24-28)$ & $25(23-28)$ & $0.232^{\ddagger}$ \\
\hline Median time on cART (25th - 75th), months & $18(2-55)$ & $18(2-54)$ & $16(2-75)$ & $0.862^{\ddagger}$ \\
\hline \multicolumn{5}{|l|}{ Marital status, $n(\%)$} \\
\hline Married & $128(73)$ & $108(73)$ & $20(74)$ & Reference \\
\hline Single & $47(27)$ & $40(27)$ & $7(26)$ & $0.913^{\varsigma}$ \\
\hline \multicolumn{5}{|l|}{ Laboratory and clinical parameters } \\
\hline Median CD4+ count (25th - 75th), cells $/ \mu \mathrm{L}$ & $377(198-511)$ & $405(228-514)$ & $209(50-405)$ & $0.012^{\star *}$ \\
\hline Median VL (25th - 75th), copies/mL & $97(10-1400)$ & $49(10-686)$ & $3033(76-87604)$ & $<0.001^{* *}$ \\
\hline \multicolumn{5}{|c|}{$\begin{array}{l}\text { CMV }=\text { cytomegalovirus; } \mathrm{SD}=\text { standard deviation; } 25 \text { th }-75 \text { th }=\text { interquartile range; } \mathrm{BMI}=\text { body mass index; cART }=\text { combination antiretroviral therapy; } \mathrm{VL}=\mathrm{HIV} \text { viral load. } \\
{ }^{*} \text { Statistically significant. } \\
t \text { t-test. } \\
\text { "Mann-Whitney rank sum test. } \\
\text { " } \chi^{2} \text { test. }\end{array}$} \\
\hline
\end{tabular}


(25th - 75th percentile) 3033 (76 - 87 604) copies/mL) compared with CMV-uninfected women (median (25th - 75th percentile) 49 (10 686) copies/mL) (Fig. 2). A stepwise multivariate logistic regression analysis was employed to account for the possible confounding factors, including maternal age, gestational age, BMI, gravidity, HIV viral load, CD4+ T-lymphocyte and plasma EFV concentration on plasma CMV DNA status (Table 2). Plasma EFV concentration and HIV viral load were the significant determinants of plasma CMV DNA positivity in the final multivariate model.

\section{Association of efavirenz with drug response genotypes}

The possible role of genetic variation in CYP2B6 regarding the variability in plasma EFV concentration was determined by genotyping two polymorphisms, CYP2B6 c.516G>T and CYP2B6 c.983C $>$ T. In agreement with previous findings, plasma EFV concentrations were correlated with CYP2B6 genotypes. CYP2B6 poor-metaboliser genotypes had significantly higher plasma EFV concentrations compared with CYP2B6 extensive metabolisers. Comparisons across the genotype groups for both CYP2B6 c.516G>T

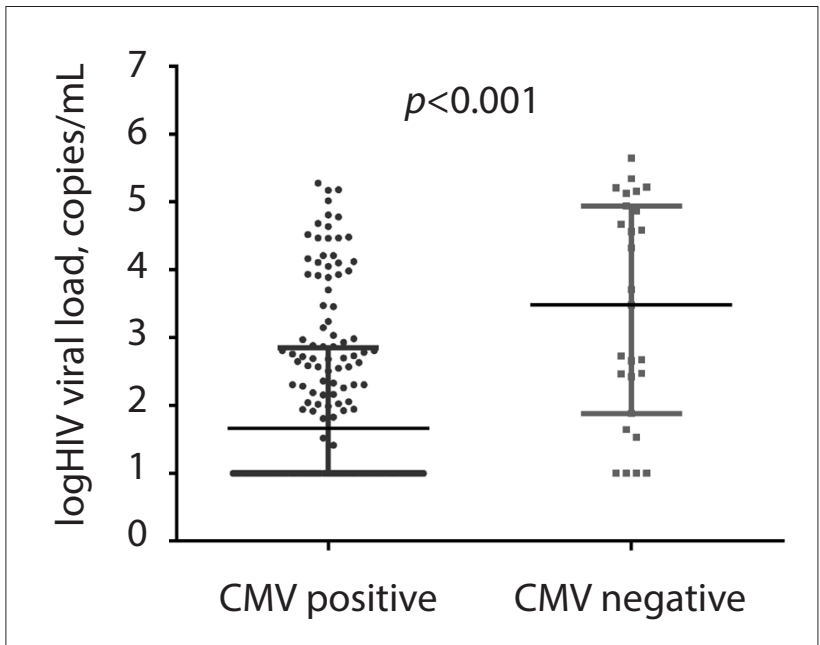

Fig. 2. Comparison of HIV viral load between CMV-infected and CMVuninfected participants. Data are presented with median and interquartile ranges. (CMV = cytomegalovirus.) and CYP2B6 c.983C $>T$ were significantly different $(p<0.001)$. For example, median plasma EFV concentrations for CYP2B6 c.516G/G, CYP2B6 c.516G/T, CYP2B6 c.516T/T, CYP2B6 c.983T/T and CYP2B6 c.983T/C were as follows: $1027 \mathrm{ng} / \mathrm{mL}, 2108 \mathrm{ng} / \mathrm{mL}$, $7074 \mathrm{ng} / \mathrm{mL}, 1695 \mathrm{ng} / \mathrm{mL}$ and $7228 \mathrm{ng} / \mathrm{mL}$, respectively.

\section{Discussion}

There is an increasing number of people living with HIV, who receive chronic cART. The biggest challenge in patient outcomes is the effect of long-term cART on susceptibility to and pathological dynamics of HIV co-infections. ${ }^{[24]}$ Even in the presence of cART, which has dramatically reduced the vertical transmission of HIV, cCMV remains a public health concern owing to late and underdiagnosis. ${ }^{[25]}$ In this study, we report a significant association between detection of plasma CMV DNA and subtherapeutic plasma EFV concentrations in pregnant women administered an EFV-based first-line cART regimen. The study also confirms the known significant association between plasma EFV concentrations and genetic variation in CYP2B6, as previously reported by Swart et al. ${ }^{[19]}$ and Lamorde et al. ${ }^{[26]}$

All participants were enrolled $>8$ hours after the EFV dose to ensure a time-point beyond the EFV absorption phase. ${ }^{[27]}$ Furthermore, all enrolled participants had been on an EFV-based regimen for $>4$ weeks, ensuring steady-state phase. However, drug adherence was reported by participants, which may have introduced bias. Assessment of adherence to ART is a major conundrum in the care of HIV patients due to potential patient misrepresentation. More effective methods of adherence assessment, such as the medication event monitoring systems (MEMS) cap, ${ }^{[28]}$ remain largely inaccessible owing to cost. The wide range of the plasma EFV concentration (250 $15931 \mathrm{ng} / \mathrm{mL}$ ) observed is indicative of the interindividual variability in exposure to therapy. Of note, $25 \%$ and $24 \%$ of participants were in the subtherapeutic and supratherapeutic groups, respectively. Regardless of patients receiving the same drug dosage, a great deal of variability in pharmacokinetics and treatment outcomes has also been documented for various drugs such as warfarin, digoxin and tacrolimus.

Several environmental, demographic and clinical factors have been implicated in the interindividual variability in response to therapy. ${ }^{[29-31]}$ However, in this study, no association between plasma EFV concentrations and demographic markers, such as maternal

Table 2. Stepwise logistic regression of clinical and demographic variables associated with cytomegalovirus DNA status

\begin{tabular}{|c|c|c|c|c|}
\hline Variables & Odds ratio & Standard error & $p$-value & $95 \% \mathrm{CI}$ \\
\hline Gestational age & 1.0 & 0.56 & 0.843 & $0.93-1.15$ \\
\hline Gravidity & 0.8 & 0.22 & 0.496 & $0.48-1.37$ \\
\hline Maternal age & 1.0 & 0.06 & 0.767 & $0.91-1.14$ \\
\hline BMI & 0.9 & 0.06 & 0.256 & $0.83-1.05$ \\
\hline $\mathrm{CD} 4+$ count $^{\dagger}$ & 0.9 & 0.03 & 0.092 & $0.99-1.00$ \\
\hline HIV viral load ${ }^{\ddagger}$ & 1.4 & 0.27 & 0.062 & $1.01-2.13$ \\
\hline EFV concentration ${ }^{*}$ & 0.4 & 0.12 & $0.002^{\star}$ & $0.22-0.72^{\star}$ \\
\hline \multicolumn{5}{|c|}{ Remove maternal age, $\mathrm{BMI}$, gestational age, count and gravidity as covariates } \\
\hline $\mathrm{CD} 4+$ count $^{\dagger}$ & 0.9 & 0.03 & 0.073 & $0.99-1.00$ \\
\hline EFV concentration ${ }^{*}$ & 1.5 & 0.12 & $0.004^{*}$ & $0.23-0.71^{*}$ \\
\hline HIV viral load ${ }^{*}$ & 0.4 & 0.26 & $0.013^{\star}$ & $0.99-2.02^{*}$ \\
\hline \multicolumn{5}{|l|}{ Remove CD4+ count } \\
\hline Plasma EFV concentration ${ }^{*}$ & 0.6 & 0.14 & $0.024^{*}$ & $0.38-0.93^{*}$ \\
\hline HIV viral load ${ }^{*}$ & 1.6 & 0.26 & $0.005^{\star}$ & $1.15-2.18^{\star}$ \\
\hline
\end{tabular}


age, BMI, duration on cART and gestational age (Suppl. Table 1) was observed. Considering the distribution of plasma EFV concentration among the participants, with $25 \%$ in the subtherapeutic group and at risk of viral replication, the $400 \mathrm{mg}$ once-daily dose currently recommended partly because of data from the ENCORE1 (efficacy of $400 \mathrm{mg}$ efavirenz $\mathrm{v}$. the standard $600 \mathrm{mg}$ dose in HIV-infected antiretroviral-naive adults) study, may not be ideal. This study concluded that an EFV dose reduction from $600 \mathrm{mg}$ to $400 \mathrm{mg}$ once daily would result in optimal therapy ${ }^{[32]}$ However, considering findings from the current study, further reduction of the standard dose would further predispose patients in the subtherapeutic group to viral replication and risk of developing co-infections. Perhaps, genotype-assisted dosing adjustments are ideal. It is worth noting that the ENCORE1 study was not carried out in pregnant women, and therefore may not be appropriately extrapolated to such women. Metabolic aberrations that occur during pregnancy could potentially impact drug metabolism. For example, in pregnancy, there is a general decrease in albumin content $(\sim 30 \%)$ in the third trimester, resulting in a reduced albumin availability. ${ }^{[33]}$ Albumin binds EFV; therefore, increased unbound EFV may increase penetration of HIV-infected tissue cells. Furthermore, most of the results from the ENCORE1 study are premised on a different population grouping compared with participants in our study, who were predominantly of African ancestry. ${ }^{[34]}$

Understanding factors that influence exposure to drugs is important, especially during pregnancy, when there is a risk of transmission of antenatal co-infections to the developing fetus. The proportion of decreased plasma EFV concentrations and CMV viral load in the current study is a cause for concern. It is known that subtherapeutic cART concentrations sustain HIV replication. CMV and HIV have previously been shown to coactivate each other. ${ }^{[35]}$ The current study further supports previous reports, which hypothesise that HIV flourishes in patients with low cART concentrations, which in turn supports CMV replication, resulting in higher CMV viral loads. We also hypothesise the contribution of CMV to poor health and hearing impairment experienced by HIV-exposed children born to mothers with high HIV viral loads. To further support the relationship between HIV replication and the likelihood of CMV infection, we found a significant association between increased HIV viral load and CMV positivity.

The observed CMV DNA positivity in this study is most likely due to CMV reactivation during pregnancy, considering the ubiquitous nature of CMV in resource-poor settings. Taylor et al. ${ }^{[36]}$ reported that cART acts by disrupting the HIV life cycle, thereby arresting viral replication. When there is insufficient cART in circulation, HIV replicates, thereby further weakening the immune system. Latent viruses such as CMV are reactivated in the setting of compromised immunity and chronic immune activation. Hence, subtherapeutic EFV concentrations predispose to CMV reactivation, which, if occurring in pregnancy, may have adverse health outcomes on the growing fetus and/or neonate. CMV and HIV have previously been found to co-activate each other in vitro and have been described as 'partners in crime', with CMV viral shedding thereby activating the immune system, which then stimulates HIV replication. ${ }^{[13]}$ We therefore hypothesise that the subtherapeutic EFV concentrations predisposed the pregnant women to CMV infection and perhaps reactivation. In view of these findings, dose reduction should only be implemented in view of an individual's genotype to avoid subtherapeutic drug concentrations that may predispose to co-infections.

Data in this cohort of pregnant women show that CYP2B6 c.516T and CYP2B6 c.983C variants are good correlates of plasma EFV concentrations. These findings argue for pharmacogenetic-guided EFV dosing. Previous studies have reported similar findings, such as a reduced activity of the CYP2B6 enzyme due to the CYP2B6 c.516G $>T$ and CYP2B6 c.983T>C SNPs. ${ }^{[5,18,26]}$ Consequently, carriers of the CYP2B6 c.516T and CYP2B6 c.983C variants have been associated with high CYP2B6 substrate drug concentrations, which increase with copy number (i.e. CYP2B6 $\quad$ c.516T/T $\gg>C Y P 2 B 6$ c. $516 G / T \gg$ >CYP2B6 $\quad$ c. $516 G / G, \quad C Y P 2 B 6 \quad c .983 C / C>>>C Y P 2 B 6$ c. $983 C / T>>C Y P 2 B 6$ c. $983 T / T)$. The CYP2B6 c.516T and CYP2B6 c. $983 \mathrm{C}$ alleles both result in reduced enzyme function, leading to ineffective metabolism of EFV and high drug concentrations.

\section{Conclusions}

We conclude that subtherapeutic cART concentrations may predispose to viral replication and co-infections such as CMV. We also confirm that polymorphisms in the CYP2B6 gene are potential pharmacokinetic determinants of EFV and as such should be considered for dose adjustment.

\section{Declaration. None.}

Acknowledgements. Drug concentration analysis for the project was supported by Grant No. D43TW010313 from the Fogarty International Center, USA. The content is solely the responsibility of the authors and does not necessarily represent the official views of the Fogarty International Center or the National Institutes of Health, USA. Genetic characterisation for this project was supported by the National Research Foundation of South Africa (SA), the University of Cape Town, SA, and Letten Research Foundation House, Harare, Zimbabwe. The authors would like to pay tribute to the late Professor Babill Stray-Pederson, who passed on (24 April 2019). May her soul rest in peace.

Author contributions. DM conceived the ideas, carried out laboratory experiments, analysed data and drafted the manuscript; GM and CM coconceived the ideas, co-supervised the pharmacology components and reviewed the manuscript draft; KM reviewed the manuscript draft, and assisted with laboratory experiments and data analysis; $\mathrm{CD}$ conceived the ideas, supervised all components as principal investigator and reviewed the manuscript draft.

Funding. Grant No. D43TW010313 from the Fogarty International Center, USA, National Research Foundation of SA, University of Cape Town, SA, and Letten Foundation Research House of Norway.

Conflicts of interest. None.

1. Taha TE. Mother-to-child transmission of HIV in sub-Saharan Africa: Past, present and future challenges. Life Sci 2011;88(21-22):917-921. https://doi.org/10.1016/j.lfs.2010.09.031

2. World Health Organization. Use of Antiretroviral Drugs for Treating Pregnant Women and Preventing World Health Organization. Use of Antiretroviral Drugs for Treating Pregnant Women and Preventing
HIV Infection in Infants. Geneva: WHO, 2012. https://www.who.int/hiv/pub/mtct/programmatic_

3. Rosala-Hallas A, Bartlett JW, Filteau S. Growth of HIV-exposed uninfected, compared with HIV Rosala-Hallas A, Bartlett JW, Filteau S. Growth of HIV-exposed uninfected, compared with HIV-
unexposed, Zambian children: A longitudinal analysis from infancy to school age. BMC Pediatr 2017;17(1):80. https://doi.org/10.1186/s12887-017-0828-6

4. Slogrove A, Reikie B, Naidoo S, et al. HIV-exposed uninfected infants are at increased risk for severe infections in the first year of life. J Trop Pediatr 2012;58(6):505-508. https://doi.org/10.1093/tropej fms019

5. Neary M, Owen A. Pharmacogenetic considerations for HIV treatment in different ethnicities: An update. Expert Opin Drug Metab Toxicol 2017;13(11):1169-1181. https://doi.org/10.1080/17425255 .2017.1391214

6. Ramokolo V, Goga AE, Lombard C, Doherty T, Jackson DJ, Engebretsen IM. In utero ART exposure and birth and early growth outcomes among HIV-exposed uninfected infants attending immunization services: Results from national PMTCT surveillance, South Africa. Open Forum Infect Dis 2017;4(4):ofx187. https://doi.org/10.1093/ofid/ofx187

7. Dah,44):ofx 187. https://doi.org/10.1093/ofid/ors 187 . Dahourou DL, Gautier-Lafaye C, Teasdale CA, et al. Transition from paediatric to adult care of
adolescents living with HIV in sub-Saharan Africa: Challenges, youth-friendly models, and outcomes. adolescents living with HIV in sub-Saharan Africa: Challenges, youth-friendly m

8. Diale Q, Pattinson R, Chokoe R, Masenyetse L, Mayaphi S. Antenatal screening for hepatitis B virus Diale Q, Pattinson R, Chokoe R, Masenyetse L, Mayaphi S. Antenatal screening for hepatitis B virus
in HIV-infected and uninfected pregnant women in the Tshwane district of South Africa. S Afr Med J 2015;106(1):97-100. https://doi.org/10.7196/SAMJ.2016.v106i1.9932 
9. Patil S, Bhosale R, Sambarey P, et al. Impact of maternal human immunodeficiency virus infection on pregnancy and birth outcomes in Pune, India. AIDS Care 2011;23(12):1562-1569. https://doi.org/10.1 080/095401212011.579948

10. Alvarado-Hernández DL, Benítez-Sánchez A, Rodríguez-Cuevas JS, et al. Killer-cell immunoglobulinlike receptors and cytomegalovirus reactivation during late pregnancy. Int J Immunogenet 2016:43(4):189-199. https://doi.org/10.1111/iji.1227

11. Lanzieri TM, Chung W, Flores M, et al. Hearing loss in children with asymptomatic congenital cytomegalovirus infection. Pediatrics 2017;139(3). https://doi.org/10.1542/peds.2016-2610

2. Madrid L, Varo R, Maculuve S, et al. Congenital cytomegalovirus, parvovirus and enterovirus infection in Mozambican newborns at birth: A cross-sectional survey. PLoS ONE 2018;13(3):e0194186. https://doi. org/10.1371/journal.pone.0194186

13. Filteau S, Rowland-Jones S. Cytomegalovirus infection may contribute to the reduced immune function growth, development, and health of HIV-exposed, uninfected African children. Front Immunol 2016;7:257. https://doi.org/10.3389/fimmu.2016.00257

14. Grinsztejn B, Hosseinipour MC, Ribaudo HJ, et al. Effects of early versus delayed initiation of antiretroviral treatment on clinical outcomes of HIV infection: Results from the phase 3 HPTN 052 randomised controlled trial. Lancet Infect Dis 2014;14(4):281-290. https://doi.org/10.1016/S14733099(13)70692-3

15. Zash R, Jacobson DL, Diseko M, et al. Comparative safety of antiretroviral treatment regimens in pregnancy. JAMA Pediatr 2017:171(10):e172222. https://doi.org/10.1001/jamapediatrics.2017.2222

16. Robarge JD, Metzger IF, Lu J, et al. Population pharmacokinetic modeling to estimate the contribution of genetic and non-genetic factors to efavirenz disposition. Antimicrob Agents Chemother 2017;61(1). https:/doiog/10.1128/AAC 01813-16

17. Polo M, Alegre F, Funes HA, et al. Mitochondrial (dys)function - a factor underlying the variability of efavirenz-induced hepatotoxicity? Br J Pharmacol 2015;172(7):1713-1727. https://doi.org/10.1111/ bph. 13018

18. Haas DW, Kwara A, Richardson DM, et al. Secondary metabolism pathway polymorphisms and plasma efavirenz concentrations in HIV-infected adults with CYP2B6 slow metabolizer genotypes. J Antimicrob Chemother 2014;69(8):2175-2182. https://doi.org/10.1093/jac/dku110

19. Swart M, Skelton M, Ren Y, Smith P, Takuva S, Dandara C. High predictive value of CYP2B6 SNPs for steady-state plasma efavirenz levels in South African HIV/AIDS patients. Pharmacogenet Genomic 2013;23(8):415-427. https://doi.org/10.1097/FPC.0b013e328363176

20. Ministry of Health and Child Care, Zimbabwe. Zimbabwe National Guidelines on HIV Testing and Counselling. Harare: Ministry of Health and Child Care Zimbabwe, 2014. https://aidsfree.usaid.gov (accessed 18 July 2019).

21. Mhandire D, Lacerda M, Castel S, et al. Effects of CYP2B6 and CYP1A2 genetic variation on nevirapine plasma concentration and pharmacodynamics as measured by CD4 cell count in Zimbabwean HIVinfected patients. OMICS 2015;19(9):553-562. https://doi.org/10.1089/omi.2015.0104

22. Harris PA, Taylor R, Thielke R, Payne J, Gonzalez N, Conde JG. Research electronic data captur (REDCap) - a metadata-driven methodology and workflow process for providing translational research informatics support. J Biomed Inform 2009;42(2):377-381. https://doi.org/10.1016/j.jbi.2008.08.010
23. Marzolini C, Telenti A, Decosterd LA, Greub G, Biollaz J, Buclin T. Efavirenz plasma levels can predict treatment failure and central nervous system side effects in HIV-infected patients. AIDS $2001: 15(1): 71-75$

24. Gianella S, Letendre S. Cytomegalovirus and HIV: A dangerous pas de deux. J Infect Dis 2016:214(Suppl 2):S67-S74, https:///doi.org/10.1093/infdis/iiw217

25. Manicklal S, Emery VC, Lazzarotto T, Boppana SB, Gupta RK. The 'silent' global burden of congenital cytomegalovirus. Clin Microbiol Rev 2013;26(1):86-102. https://doi.org/10.1128/CMR.00062-12

26. Lamorde M, Wang X, Neary M, et al. Pharmacokinetics, pharmacodynamics, and pharmacogenetics of efavirenz $400 \mathrm{mg}$ once daily during pregnancy and post-partum. Clin Infect Dis 2018;67(5):785-790. https://doi.org/10.1093/cid/ciy161

27. Bednasz CJ, Venuto CS, Ma Q, et al. Efavirenz therapeutic range in HIV treatment-naive participants. Ther Drug Monit 2017;39(6):596-603. https://doi.org/10.1097/FTD.0000000000000443

28. Claxton AJ, Cramer J, Pierce C. A systematic review of the associations between dose regimens and medication compliance. Clin Ther 2001;23(8):1296-1310.

29. Swaminathan S, Ramachandran G, Agibothu Kupparam HK, et al. Factors influencing plasma nevirapine levels: A study in HIV-infected children on generic antiretroviral treatment in India. J Antimicrob Chemother 2011;66(6):1354-1359. https://doi.org/10.1093/jac/dkr075

30. Jaakkola S, Nuotio I, Kiviniemi TO, Virtanen R, Issakoff M, Airaksinen KEJ. Incidence and predictors of excessive warfarin anticoagulation in patients with atrial fibrillation - the EWA study. PLoS ONE 2017;12(4). https://doi.org/10.1371/journal.pone.0175975

31. Bienczak A, Denti P, Cook A, et al. Plasma efavirenz exposure, sex, and age predict virological response in HIV-infected African children. J Acquir Immune Defic Syndr 2016;73(2):161-168. https://doi.org/10.1097/QAI.0000000000001032

32. Dickinson L, Amin J, Else L, et al. Pharmacokinetic and pharmacodynamic comparison of oncedaily efavirenz (400 mg vs. $600 \mathrm{mg}$ ) in treatment-naive HIV-infected patients: results of the ENCORE1 study. Clin Pharmacol Ther 2015;98(4):406-416. https://doi.org/10.1002/cpt.156

33. Perucca E, Ruprah M, Richens A. Altered drug binding to serum proteins in pregnant women: Therapeutic relevance. J R Soc Med 1981;74(6):422-426. https://doi.org/10.1177/014107688107400606

34. Shah RR, Gaedigk A. Precision medicine: Does ethnicity information complement genotypebased prescribing decisions? Ther Adv Drug Saf 2018;9(1):45-62. https://doi.org/10.1177/ 2042098617743393

35. Freeman ML, Lederman MM, Gianella S. Partners in Crime: The role of CMV in immune dysregulation and clinical outcome during HIV infection. Curr HIV/AIDS Rep 2016;13(1):10-19. (10)

36. Taylor BS, Olender SA, Tieu H-V, Wilkin TJ. CROI 2016: Advances in antiretroviral therapy. Top Antivir Med 2016;24(1):59-81.

Accepted 10 September 2019

Supplementary Table 1. Stepwise multivariate linear regression of clinical and demographic variables that predict plasma efavirenz concentration

\begin{tabular}{|c|c|c|c|c|}
\hline Variables & Coefficient & Standard error & $p$-value & 95\% CI \\
\hline CYP2B6 c.516 G/T & 0.67 & 0.13 & $<0.001^{*}$ & $0.42-0.93$ \\
\hline CYP2B6 c.516 T/T & 1.58 & 0.15 & $<0.001^{*}$ & $1.28-1.90$ \\
\hline CYP2B6 c.983 T/C & 0.88 & 0.17 & $<0.001^{\star}$ & $0.55-1.21$ \\
\hline Gestational age & -0.01 & 0.012 & 0.307 & $-0.04-0.01$ \\
\hline Gravidity & -0.01 & 0.06 & 0.821 & $-0.12-0.11$ \\
\hline Maternal age & 0.01 & 0.013 & 0.577 & $-0.02-0.032$ \\
\hline BMI & -0.004 & 0.014 & 0.767 & $-0.03-0.03$ \\
\hline $\mathrm{CD} 4+$ count $^{\dagger}$ & -0.01 & 0.01 & 0.133 & $-0.001-0.0000$ \\
\hline HIV viral load & -0.26 & 0.04 & $<0.001^{*}$ & $-0.35-0.18$ \\
\hline \multicolumn{5}{|c|}{ Remove gravidity and gestational age as covariates } \\
\hline CYP2B6 c.516 G/T & 0.68 & 0.13 & $<0.001^{*}$ & $0.42-0.94$ \\
\hline CYP2B6 c.516 T/T & 1.59 & 0.15 & $<0.001^{*}$ & $1.29-1.89$ \\
\hline CYP2B6 c.983 T/C & 0.91 & 0.17 & $<0.001^{*}$ & $0.58-1.23$ \\
\hline Maternal age & 0.01 & 0.01 & 0.612 & $-0.02-0.03$ \\
\hline BMI & -0.01 & 0.14 & 0.654 & $-0.03-0.02$ \\
\hline $\mathrm{CD} 4+$ count $^{\dagger}$ & -0.01 & 0.01 & 0.128 & $-0.001-0.00001$ \\
\hline HIV viral load & -0.25 & 0.04 & $<0.001^{*}$ & $-0.35-0.17$ \\
\hline \multicolumn{5}{|c|}{ Remove BMI, maternal age and CD4+ count } \\
\hline CYP2B6 c.516 G/T & 0.68 & 0.13 & $<0.001^{\star}$ & $0.43-0.94$ \\
\hline CYP2B6 c.516 T/T & 1.59 & 0.15 & $<0.001^{*}$ & $1.30-1.90$ \\
\hline СYР2B6 c.983 T/C & 0.91 & 0.16 & $<0.001^{\star}$ & $0.60-1.23$ \\
\hline HIV viral load & -0.23 & 0.04 & $<0.001^{*}$ & $-0.35-0.18$ \\
\hline
\end{tabular}

